\title{
A Sufficient Criterion to Choose Quadratic Lyapunov Functions for Nonlinear Systems ${ }^{1}$
}

L.G.S. LONGHI 2 , Departamento de Engenharia Química, Pontifícia Universidade Católica do Rio Grande do Sul, Avenida Ipiranga, 6681, Building 30, 90619-900 Porto Alegre, RS, Brazil

A.R. SECCHI ${ }^{3}$, Departamento de Engenharia Química, Universidade Federal do Rio Grande do Sul, Rua Eng. Luiz Englert s/n, 90050-170 Porto Alegre, RS, Brazil.

\begin{abstract}
A sufficient criterion to determine sign definiteness regions for multivariable scalar functions is presented. This criterion is used as a constraint in the formulation of an optimization problem to maximize the estimate of the domain of attraction for asymptotically stable nonlinear systems using quadratic forms as Lyapunov functions. Two examples whose estimates are as good as the ones obtained from more complex methods reported in the literature illustrate the usefulness of the main results.
\end{abstract}

Keywords: Lyapunov theory; domain of attraction; stability; quadratic forms

\section{Introduction}

This work deals with the quantitative stability analysis of nonlinear autonomous dynamical systems. Consider a smooth system described by (1.1)

$$
\frac{d x(t)}{d t}=\dot{x}=f(x),
$$

where $x \in \Re^{n}$ is the $\mathrm{n}$-dimensional vector of the state variables and the origin is the steady state solution of interest, $f(0)=0$. It is well known in the control and systems literature that the local asymptotic stability is not sufficient to guarantee the stability of a real system [1]. Many times, the domain of attraction (DA) is so small that it is no useful for practical purposes. Consequently, the attainment of quantitative results about the size of this domain is very important for a practical stability analysis. As the exact determination of DA for general nonlinear systems is usually a hard task, simpler estimates of that region are commonly well accepted for

\footnotetext{
${ }^{1}$ Trabalhos apresentados no CNMAC.

2llonghi@pucrs.br

3 arge@enq.ufrgs.br
} 
quantitative analysis. The simplest estimates consider quadratic forms as Lyapunov function candidates:

$$
V(x)=x^{T} P x .
$$

The determination of subsets of the DA for nonlinear systems using the Lyapunov stability theory results with quadratic forms is an extensively studied subject in the control literature $[2,3,4]$. The use of these forms simplifies the inequalities to be solved, and furnishes, since $\mathrm{P}$ must be positive definite, according to (1.3), conservative but useful ellipsoidal estimates for DA.

$$
D(\rho)=\left\{x: V(x)<\rho^{2} \text { and } \dot{V}(x)<0\right\} .
$$

The usual approach to estimate DA with quadratic Lyapunov functions consists on a two steps procedure [4]:

(i) A Lyapunov matrix $P(>0)$ is chosen according to some criteria.

(ii) The optimal estimate for this form $\left(\rho_{\text {opt }}\right)$ is computed by solving the following optimization problem:

$$
\begin{aligned}
& \rho_{\text {opt }}=\quad \inf _{x \in \Re^{n}} \quad x^{T} P x . \\
& \text { subject to } \dot{V}(x)=0
\end{aligned}
$$

This usual approach has two drawbacks:

1. The Lyapunov matrix $\mathrm{P}$ is chosen without regarding the size of DA.

2. As the optimization problem (1.4) is in general nonconvex, its solution may lead to a local minimum and consequently to a fake DA;

One of the main reasons why it is not possible to choose a Lyapunov matrix P to directly maximize the DA is the absence of sign definiteness criteria for multivariable scalar functions. In this work, a sufficient criterion to determine subsets of the sign definiteness region for multivariable scalar functions is presented. Based on this criterion, a methodology to:

1. choose the matrix $P>0$ to maximize the estimate of the sign definiteness region of $\dot{V}(x)$ and

2. formulate a single step optimization problem to directly maximize the estimate of the DA, considering the sufficient criterion for the negativeness of $\dot{V}(x)$ as an inequality constraint,

is proposed.

\section{Main results}

The problem of determining the asymptotic stability for system (1.1) with quadratic Lyapunov functions can be viewed as the problem of finding a positive scalar function:

$$
V(x)=x^{T} P x>0,
$$


which satisfies a strict inequality for its time derivative:

$$
\frac{d V(x)}{d t}=\frac{\partial V(x)}{\partial x} \dot{x}=\frac{\partial V(x)}{\partial x} f(x)<0
$$

in some neighborhood of the origin.

Although the famous Sylvester criteria provides simple necessary and sufficient conditions to verify the global positivity of quadratic forms, there are no criteria to check the sign definiteness of general nonlinear functions. Here, to obtain a criterion that could be used to estimate the sign definiteness region of (2.2), it is presented in Theorem 1 a recent result concerning the sign definiteness of multivariable scalar functions [5], which requires the Definition 1 before.

Definition 1. The real local region, $\Omega \subset \Re^{n}$, of a multivariable function $y(x): \Re^{n} \rightarrow C^{n}$, is the set composed by the intersection of the subsets of $\Re^{n}$ where each element of $x$ can assume values such that $y(x)$ belongs to a subset $\Psi \subset \Re^{n}$ and $y \neq 0$ unless $x=0$.

Theorem 1. Let $\dot{V}(x)$ be a scalar function of $n$ real variables. If the quadratic form representation of $\dot{V}(x)$ is $y^{T}(x) P_{V} y(x)$, where the components of $y$ are functions of $x \in \Omega$ and $P_{V}$ is a symmetric real matrix obtained directly from $\dot{V}(x)$, then a sufficient condition for the local negativity of $\dot{V}(x)$ is that $P_{V}$ be a negative definite matrix. Consequently, $\Omega$ is the local region where $\dot{V}(x)$ is negative.

\section{Proof:}

Sufficiency - If $\dot{V}(x)$ can be written as a quadratic form, $y^{T}(x) P_{V} y(x)$, where $y(x)$ are nonlinear functions of $x$ and $P_{V}$ is a real symmetric matrix, it is a well known result from linear algebra [6] that it also can be written in new coordinates $z=U^{T} y$ as

$$
\dot{V}(x)=z(y(x))^{T} \Lambda z(y(x))=z^{T}(x) \Lambda z(x),
$$

where $U$ is an orthonormal matrix whose columns, $u_{j}$, are the eigenvectors of $P_{V}$, and $\Lambda$ is a diagonal matrix having the same eigenvalues as $P_{V}$. Then, $\dot{V}(x)$ can be rewritten as:

$$
\dot{V}(x)=\lambda_{1} z_{1}^{2}+\lambda_{2} z_{2}^{2}+\ldots+\lambda_{n} z_{n}^{2},
$$

with

$$
z_{i}=\left(u_{i 1} y_{1}(x)+u_{i 2} y_{2}(x)+\ldots+u_{i n} y_{n}(x)\right), \quad \text { for } \quad i=1 \text { to } n .
$$

This orthonormal coordinate transformation maintains the norm and the inner product of $P_{V}$ and, consequently, conclusions about the sign of $\dot{V}(x)$ are also maintained.

Because all the eigenvalues of a real symmetric matrix belong to the real field, if all the eigenvalues of $P_{V}$ are negative (i.e., the matrix is negative definite) $\dot{V}(x)$ is negative definite if, for any nonzero $x$ :

(i) $z_{j}^{2}(x) \geq 0$ (for $j=1$ to $n$ ), and 
(ii) $z_{j}^{2}(x)>0$ for at least one $j$.

Now, it must be noted that the conditions (i)-(ii) are only satisfied in the real local region of $y$, that is the region where the sign of $\dot{V}(x)$ is negative. Then, the negativity of $P_{V}$ implies $\dot{V}(x)$ is negative definite inside that region.

Not necessity - Consider a positive function having a quadratic form representation, $y^{T}(x) P_{V} y(x)$, with $P_{V}$ negative definite. As the quadratic form representation is arbitrary, there is no restriction to add a new nonzero element to vector $y$. There is no loss of generality if this new element has no influence on the original negative function because the components of $y$ are not necessarily independent. If this occurs, despite the fact the function is negative, the new eigenvalue corresponding to this new element will be null. So, the negativeness of $\dot{V}(x)$ does not imply that $P_{V}$ is negative definite and theorem 1 is only a sufficient condition. This also proves that the negative semi-definiteness of $P_{V}$ does not imply in $\dot{V}(x) \leq 0$.

Now, with the help of theorem 1 , it is possible to choose the matrix $P$ to maximize the size of the sufficient negative region of $\dot{V}(x)$ while maintaining $P>0$ and $P_{V}<0$.

Optimiz. problem 1 - Substitute the quadratic form $V(x)=x^{T} P x$ in $\dot{V}(x)=$ $\frac{\partial V(x)}{\partial x} f(x)$. Write this last equation as a quadratic form representation:

$$
\dot{V}(x)=y^{T}(x) P_{V} y(x)
$$

where $P$ and $P_{V}$ are symmetric real matrices obtained directly from the coefficients of $V(x)$ and $\dot{V}(x)$, respectively. Let $\alpha_{i}$ be the parameters of $y(x)$ which define the negativity region of $\dot{V}(x)$. Choose the parameters of $V(x)$, i.e. the matrix $P$, and $y(x)$, i.e. the parameters $\alpha_{i}$, in a way to maximize the sufficient negativeness region of $\dot{V}(x)$ subject to the constraints $P>0$ and $P_{V}<0$.

Although this optimization problem can provide an appealing approach to choose $P$, it needs a second step to find the DA given by (1.4) - determining the maximum of $V(x)$ inside the estimated negativity region of $\dot{V}(x)$. As this second step still is in general a nonconvex one, it can lead to local minima and consequently to a false estimate of DA. Moreover, it is necessary to derive a non-trivial analytical equation describing the negativity region of $\dot{V}(x)$ with $P$ and $\alpha_{i}$ as variables. Note that the parameters $\alpha_{i}$ give additional degree of freedom to the optimization problem to maximize the negativity region of $\dot{V}(x)$.

So, alternatively, it is more interesting to formulate an optimization problem to directly maximize the size of the ellipsoidal domain of attraction using the same quadratic Lyapunov function. Basically, this problem is the same optimization problem 1 but, instead of maximizing the negativeness region of $\dot{V}(x)$, the objective is to maximize the ellipsoid region defined by $V(x)=x^{T} P x=1$. As the volume of such ellipsoid region is proportional to the inverse of the products of the eigenvalues of $P$, the objective of the problem can be posed as the minimization of the product of the eigenvalues of $P$ (or equivalently, the determinant of $P$ ). This modified 
problem also needs an additional constraint informing that the ellipsoid $V(x)=1$ is located on the border of the sufficient negativity region. This equality constraint must link the matrix $P$ to the parameters $\alpha_{i}$. If an appropriate choice of $y(x)$ is done, this can result in a simple problem to be solved.

The one-step optimization problem, despite being a very general approach to maximize an ellipsoidal DA with a quadratic Lyapunov function, sometimes, can be very complex, and even a intractable problem. In that cases, a judicious choice for the quadratic form representation of $\dot{V}(x)$ can be very important to simplify the problem to be solved. In fact, the heart of the method is the representation of $\dot{V}(x)$, and different choices for (2.6) can be exploited to improve the estimate of the DA. It also must be noted that this one step optimization problem, different from (1.2), does not have the troublesome of finding false DA because the positivity of $V(x)$ and the negativity of $\dot{V}(x)$ are included as constraints in the problem formulation. Consequently, these sign definiteness conditions are assured to be satisfied if the problem has a feasible solution. As a final remark, the maximization of DA is still a nonconvex problem. If a global optimization algorithm is not used, a good choice for the starting solution $P_{0}$ is fundamental to improve the estimated DA. Davison and Kurak [2] used an approach that try to diminish the eccentricity of $P$ but other approaches, like the branch and bound algorithms [7], can be explored to find the global maximum of the DA. The examples presented in the next section will illustrate the use of the two optimization problem. Both examples were solved by utilizing a multishoot approach coupled with a deterministic optimization algorithm.

\section{Examples}

The objective of this section is to show how the results presented in section 2 can be used. We consider two mathematical dynamical systems. The first one aims to illustrate the application of the developed methodology to a classical and, consequently, well studied example. The next one was developed by the authors to illustrate a situation where the maximization of the negativity region of $\dot{V}(x)$ may result in unpractical estimates for the DA. In those situations, it is necessary to consider the direct DA maximization, as suggested in the previous section.

\subsection{Example 1 - Van der Pol oscillator}

Consider the classical Van der Pol system (3.1)

$$
\dot{x}=\left(\begin{array}{c}
\dot{x}_{1} \\
\dot{x}_{2}
\end{array}\right)=\left(\begin{array}{c}
x_{2} \\
x_{1}-\left(1-x_{1}^{2}\right) x_{2}
\end{array}\right)
$$

and the quadratic form

$$
V(x)=x^{T} P x=a x_{1}^{2}+b x_{1} x_{2}+c x_{2}^{2}
$$

as the Lyapunov candidate function. Its time derivative is given by

$$
\dot{V}(x)=b x_{1}^{3} x_{2}+2 c x_{1}^{2} x_{2}^{2}+b x_{1}^{2}+(-2 a-b+2 c) x_{1} x_{2}+(-b-2 c) x_{2}^{2} .
$$


If (3.3) is rewritten as the quadratic form representation

$$
\dot{V}(x)=\left[\begin{array}{c}
x_{1} \\
x_{2} \\
\sqrt[2]{\alpha_{1}-b x_{1} x_{2}-2 c x_{2}^{2}} x_{1}
\end{array}\right]^{T} P_{V_{1}}\left[\begin{array}{c}
x_{1} \\
x_{2} \\
\sqrt[2]{\alpha_{1}-b x_{1} x_{2}-2 c x_{2}^{2}} x_{1}
\end{array}\right],
$$

where $P_{V_{1}}=\left[\begin{array}{ccc}b+\alpha_{1} & c-a-b / 2 & 0 \\ c-a-b / 2 & -b-2 c & 0 \\ 0 & 0 & -1\end{array}\right]$,

then, according to Theorem 1, a sufficient region where $\dot{V}(x)$ has negative sign is given by: $\alpha_{1}-b x_{1} x_{2}-2 c x_{2}^{2}>0$.

Then, it is possible to solve the Optimization Problem 1 to find the matrix $P$ that maximizes this sign definiteness region, while maintaining the positivity of $P$ and the negativity of $P_{V_{1}}$ as inequality constraints. After that, the second step must be to find the minimum of $V(x)$ lying on $\alpha_{1}-b x_{1} x_{2}-2 c x_{2}^{2}=0$. The obtained negativity region and DA solving this two steps approach are shown in (3.5) and (3.6), respectively. Figure 1 shows these curves and the limit cycle which delimits the true DA for the Van der Pol system (3.1).

$$
\begin{gathered}
1.053 x_{2}^{2}-1.0008 x_{1} x_{2}<1 . \\
V(x)=2.42 x_{1}^{2}-2.37 x_{1} x_{2}+1.25 x_{2}^{2}<1 .
\end{gathered}
$$

Now, it is possible to improve this DA by reformulating the Optimization Problem 1 to directly maximize the DA. To simplify this task, it will be considered the following quadratic form representation:

$$
\dot{V}(x)=\left[\begin{array}{c}
x_{1} \\
x_{2} \\
x_{1}^{2} \\
x_{2}^{2} \\
\sqrt[2]{\alpha_{1}-V(x)} x_{1}
\end{array}\right]^{T} P_{V_{2}}\left[\begin{array}{c}
x_{1} \\
x_{2} \\
x_{1}^{2} \\
x_{2}^{2} \\
\sqrt[2]{\alpha_{1}-V(x)} x_{1}
\end{array}\right]
$$

where $P_{V_{2}}=\left[\begin{array}{ccccc}b+k \alpha_{1} & c-a-b / 2 & 0 & 0 & 0 \\ c-a-b / 2 & -b-2 c & 0 & 0 & 0 \\ 0 & 0 & -k a & \frac{b}{2}(1-k) & 0 \\ 0 & 0 & \frac{b}{2}(1-k) & c(2-k) & 0 \\ 0 & 0 & 0 & 0 & -k\end{array}\right]$.

Then, the solution of the one-step optimization problem

$$
\left[a, b, c, k, \alpha_{1}\right]=\min _{\substack{a, b, c, k, \alpha_{1} \\ \text { s.t. } P>0, P_{V_{2}}<0, \alpha_{1}=1}}\left[a c-\frac{b^{2}}{4}\right]
$$

furnishes the DA given by (3.9). 


$$
V(x)=2.415 x_{1}^{2}-2.415 x_{1} x_{2}+1.208 x_{2}^{2}<1
$$

and $k=2.4139$. The maximized DA of (3.9) is also shown in Figure 1. Note that the improvement over (3.6) is very small.

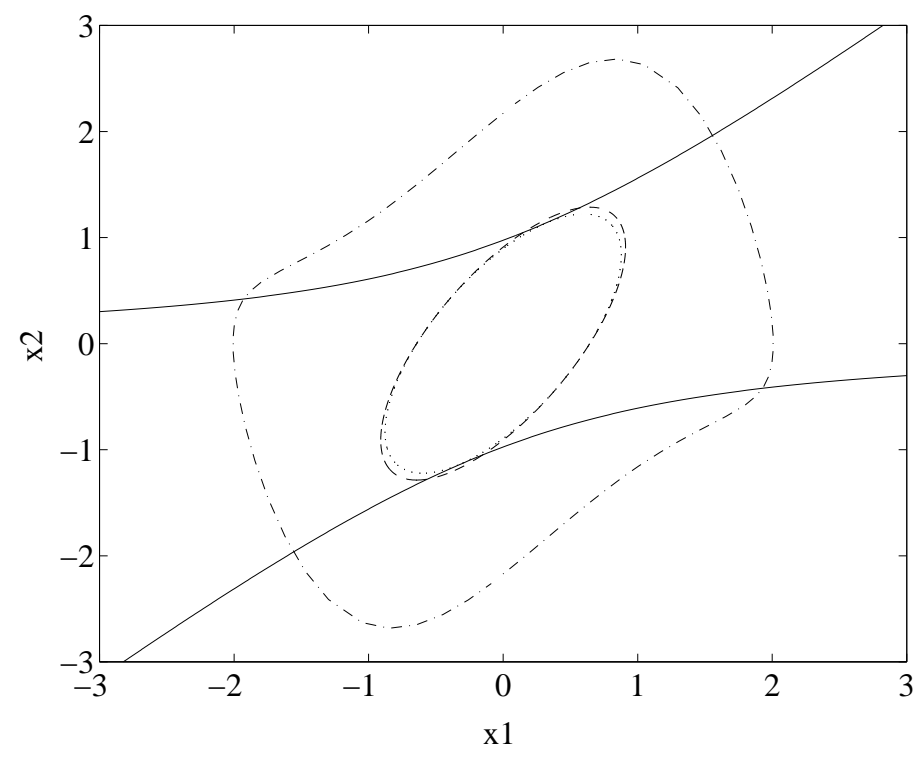

Figure 1: The sufficient negativity region of $\dot{V}(x)$ (solid line), the estimate of the DA with a quadratic form for this sufficient negativeness region (dotted line), the maximized DA with the one-step optimization problem (dashed line), and the limit cycle delimiting the true DA (dash-dotted line).

\subsection{Example 2 - A non hyperbolic one}

Consider the dynamical system (3.10) [8]

$$
\dot{x}=\left(\begin{array}{c}
\dot{x}_{1} \\
\dot{x}_{2}
\end{array}\right)=\left(\begin{array}{c}
-x_{1}+x_{1} x_{2} \\
x_{1}^{2}-x_{2}^{3}
\end{array}\right) \text {. }
$$

This system is non hyperbolic and, consequently, it is not possible to know a priori if the origin is asymptotically stable using the Lyapunov linearization method. However, by using the center manifold theory [9] it can be shown its local stability. To simplify the DA estimation, lets consider the Lyapunov function (3.11).

$$
V(x)=a x_{1}^{2}+b x_{2}^{2},
$$

whose time derivative is given by

$$
\dot{V}(x)=-2 a x_{1}^{2}+(2 a+2 b) x_{1}^{2} x_{2}-2 b x_{2}^{4}
$$


which can be rewritten as the quadratic form description (3.13)

$\dot{V}(x)=\left[\begin{array}{c}x_{1} \\ x_{2}^{2} \\ \sqrt[2]{\alpha_{1}-x_{2}} x_{1}\end{array}\right]^{T} P_{V_{3}}\left[\begin{array}{c}x_{1} \\ x_{2}^{2} \\ \sqrt[2]{\alpha_{1}-x_{2}} x_{1}\end{array}\right]$,
where $P_{V_{1}}=\left[\begin{array}{ccc}\alpha_{1}(2 a+2 b)-2 a & 0 & 0 \\ 0 & -2 b & 0 \\ 0 & 0 & -(2 a+2 b)\end{array}\right]$, where the sufficient negativity region is clearly given by: $\alpha_{1}-x_{2}>0$.

Maximizing the negativity region of $\dot{V}(x)$ subject to $a, b>0$ and $P_{V_{3}}<0$ results in the following solution: $a>0, b \rightarrow 0+$, and $\alpha_{1} \rightarrow 1$. This solution furnishes a DA given by the line segment: $\omega=\left\{x \in \Re^{2}: x_{1}=0\right.$ and $\left.-1<x_{2}<1\right\}$. Obviously, this DA has no practical value. So, if, instead of maximizing that negativity region, it is formulated an optimization problem to directly maximize the DA:

$$
\begin{gathered}
{\left[a, b, \alpha_{1}\right]=\min _{a, b, \alpha_{1}} \quad[a b]} \\
\text { s.t. } a, b, \alpha_{1}>0, P_{V_{3}}<0 \\
b \alpha_{1}^{2}-1=0
\end{gathered}
$$

the following solution can be found: $a=5.33, b=1.778$ and $\alpha_{1}=0.75$.

As the negativity region of $\dot{V}(x)$ decreased, this example shows that there is no direct relation between the size of the negativity region of $\dot{V}(x)$ and its corresponding generated DA. Figure 2 shows the estimated DA, the sufficient negativity region of $\dot{V}(x)$, and the true DA. This last curve is delimited by the stable manifolds of the saddle-points $(-1,1)$ and $(1,1)[10]$. As a final remark, if the optimization problem (3.14) is rewritten and solved considering the quadratic form representation of (3.15), where the equality constraint is given by $\alpha_{1}=1$, its solution furnishes the same DA from the former optimization problem (3.14),

$$
\begin{gathered}
\dot{V}(x)=\left[\begin{array}{c}
x_{1} \\
x_{1} x_{2} \\
x_{1}^{2} \\
x_{2}^{2} \\
\sqrt[2]{\alpha_{1}-V(x)} x_{1}
\end{array}\right]^{T} P_{V_{4}}\left[\begin{array}{c}
x_{1} \\
x_{2} x_{2} \\
x_{1}^{2} \\
x_{2}^{2} \\
\sqrt[2]{\alpha_{1}-V(x)} x_{1}
\end{array}\right], \\
\text { where } P_{V_{4}}=\left[\begin{array}{ccccc}
k \alpha_{1}-2 a & a+b & 0 & 0 & 0 \\
a+b & -k b & 0 & 0 & 0 \\
0 & 0 & -k a & 0 & 0 \\
0 & 0 & 0 & -2 b & 0 \\
0 & 0 & 0 & 0 & -k
\end{array}\right] .
\end{gathered}
$$

\section{Conclusions}

This paper presented a simple sufficient criterion to determine the sign definiteness region of multivariable scalar functions. This criterion was explored to choose 


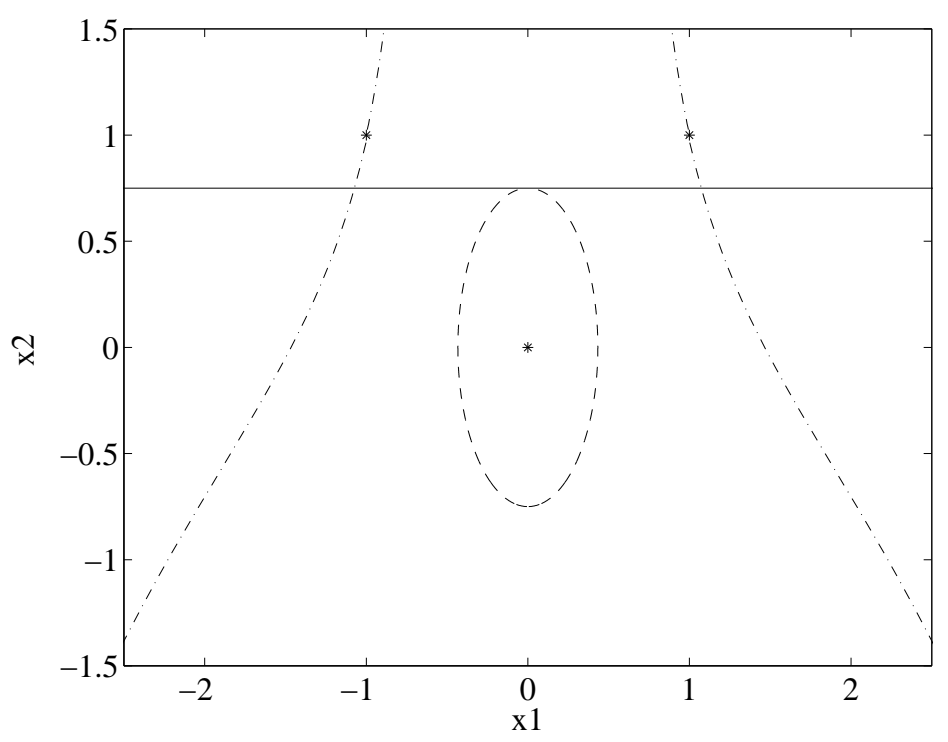

Figure 2: The sufficient negativity region of $\dot{V}(x)$ (solid line), the estimate of the DA with (3.11) as the Lapunov function (dashed line), the true DA (dotted line), and the stationary points $(*)$.

quadratic Lyapunov functions for nonlinear autonomous systems. This choice was done by maximizing the sufficient negativity region of $\dot{V}(x)$ or, preferably, by directly maximizing the DA. Two illustrative examples were presented to show the simplicity of the methodology. The main disadvantage of the method is that the rules for a suitable choice of the quadratic form representation of $\dot{V}(x)$ were not developed yet. Despite this last feature, this work could stimulate further interesting results on this subject. Nowadays, our research interest is focused on the convexification of the optimization problems, or some part of them ([11]), and on the attainment of systematic rules for chosing the quadratic forms representation for specific classes of nonlinear systems.

Resumo. Um critério suficiente para determinar regiões de sinal definido de funções escalares multivariáveis é apresentado. Este critério é utilizado como uma retrição na formulação de um problema de otimização para maximizar o domínio de atração de sistemas não-lineares assintoticamente estáveis usando formas quadráticas como funções de Lyapunov. Dois exemplos cujas estimativas são tão boas quanto as reportadas na literatura usando métodos mais complexos ilustram a utilidade do principais resultados. 


\section{References}

[1] J. La Salle and S. Lefschetz, "Stability by Liapunov's direct method", Mathematics in science and engineering 4, Academic Press, 1961.

[2] E.J. Davison and E.M. Kurak, A computational method for determining quadratic Lyapunov functions for nonlinear systems, Automatica, 7 (1971), 627-636.

[3] A. Levin, An analytical method of estimating the domain of attraction for polynomial differential equations, IEEE T. Aut. Cont., 39 (1994), 2471-2475.

[4] A. Tesi, F. Villoresi and R. Genesio, On the stability domain estimation via quadratic Lyapunov function: convexity and optimality properties for polynomial systems, IEEE Trans. Automat. Contr., 41 (1996), 1650-1657.

[5] L.G.S. Longhi, A.R. Secchi and E.L. Lima, On the positivity of multivariable scalar functions, J. Franklin Institute, 338 (2001), 509-516.

[6] E. Kreyszig, "Advanced Applied Mathematics", Prentice-Hall, 1985.

[7] C. A. Floudas, Global optimization in design and control of chemical process systems, J. Process Control, 10 (2000), 125-134.

[8] L.G.S. Longhi, A.R. Secchi and E.L. Lima, Sobre a positividade local de formas e sua aplicao teoria de Lyapunov, em "Proc. of Brazilian congress of automatica", SBA, 2000 (in portuguese).

[9] H-.D. Carr, "Applications of centre manifold theory", Springer-Verlag, 1981.

[10] H.D. Chiang, M.W. Hirsch and F.F. Wu, Stability regions of nonlinear autonomous dynamical systems, IEEE T. Autom. Contr., 33 (1988), 16-27.

[11] G. Chesi, A. Tesi, A. Vicino and R. Genesio, An LMI approach to constrained optimization with homogeneous forms, Systems and Control Letters, 42 (2001), $11-19$. 